\title{
A!
}

This is an electronic reprint of the original article.

This reprint may differ from the original in pagination and typographic detail.

Ghorbani Veshki, Farshad; Ouzir, Nora; Vorobyov, Sergiy

\section{Image Fusion using Joint Sparse Representations and Coupled Dictionary Learning}

Published in:

2020 IEEE International Conference on Acoustics, Speech, and Signal Processing, ICASSP 2020 - Proceedings

DOI:

10.1109/ICASSP40776.2020.9054097

Published: 01/05/2020

Document Version

Peer reviewed version

Please cite the original version:

Ghorbani Veshki, F., Ouzir, N., \& Vorobyov, S. (2020). Image Fusion using Joint Sparse Representations and Coupled Dictionary Learning. In 2020 IEEE International Conference on Acoustics, Speech, and Signal

Processing, ICASSP 2020 - Proceedings (pp. 8344-8348). [9054097] (Proceedings of the IEEE International Conference on Acoustics, Speech, and Signal Processing). IEEE.

https://doi.org/10.1109/ICASSP40776.2020.9054097

This material is protected by copyright and other intellectual property rights, and duplication or sale of all or part of any of the repository collections is not permitted, except that material may be duplicated by you for your research use or educational purposes in electronic or print form. You must obtain permission for any other use. Electronic or print copies may not be offered, whether for sale or otherwise to anyone who is not an authorised user. 


\title{
Image Fusion using Joint Sparse Representations and Coupled Dictionary Learning
}

\author{
Farshad G. Veshki, Nora Ouzir and Sergiy A. Vorobyov, Fellow, IEEE
}

\begin{abstract}
The image fusion problem consists in combining complementary parts of multiple images captured, for example, with different focal settings into one image of higher quality. This requires the identification of the sharpest areas in sets of input images. Recently, it was shown that coupled dictionary learning can successfully capture the relationships between highand low-resolution patches in the context of single image superresolution. In this work, to identify the sharp image patches, we propose an improved discriminative coupled dictionary learning approach using joint sparse representations in blurred and focused dictionaries. In addition, a pixel-wise processing of the boundaries (i.e., patches containing blurred and focused pixels) is proposed. The experimental results using two natural image datasets, as well as a sequence of in vivo microscopy images, show the competitiveness of the proposed method compared to stateof-the-art algorithms in terms of accuracy and computational time.
\end{abstract}

Index Terms - Image fusion, coupled dictionary learning, joint sparse representations.

\section{INTRODUCTION}

I Mage fusion is a post-processing technique that combines the relevant information from multiple images captured with different tools or parameters in a single image. Image fusion techniques seek to preserve image quality without resorting to often costly specialized optic sensors [1] $-[3]$. Due to the potential for a considerable cost reduction, image fusion has attracted increased attention in various fields, including remote sensing or medical imaging [4]-[7].

State-of-the-art image fusion methods can be grouped into two main categories: spatial and transform domain methods. The first approach relies on measures such as spatial frequency [8] and phase congruency [9] that allow the significance level of pixels (or image patches) to be evaluated. A fused image is then obtained by assigning the elements with the highest significance levels to their corresponding locations in the final image. In the second approach, the input images are transformed, and fusion is performed over the transform coefficients before generating the all-in-focus image by using inverse transform. Typical transform domain approaches use frequency-based transforms, such as wavelets [10].

One emerging image fusion approach utilizes sparse representations in dictionaries learned from the data itself [11]-[14]. These methods exploit the fact that patches of natural images can be compactly represented by a linear combination of only few atoms of an over-complete dictionary. Sparsity is then used either as a quality measure [11], or in order to learn the

The authors are with Aalto University, Dept. Signal Processing and Acoustics, FI-00076, AALTO, Finland. E-mail: farshad.ghorbaniveshki@aalto.fi, nora.ouzir@aalto.fi, svor@ieee.org desired features from the labelled training data [12]. However, these methods commonly employ a single (usually focused) dictionary. The main drawback of the single dictionary approach is that it fails to take advantage of the features in the degraded and noisy images. Specifically, the inability of properly describing degraded patches can noticeably reduce the accuracy of the sparse representation. To bypass this issue, a simultaneous learning of blurred and focused dictionaries was proposed in [14]. However, this method does not exploit the core advantage of coupled dictionary learning (CDL), which is based on a common sparse representation that expresses the correlation between blurred and focused features. Specifically, separate sparse codes are used for each dictionary, which are then averaged in order to perform fusion [14].

The CDL technique has led to state-of-the-art performance in various image processing applications [15]-[18]. CDL is designed to learn a pair of dictionaries for capturing the relationships between two correlated input data. In particular, the dictionaries are coupled, in the sense that they use common sparsity coefficients to reconstruct the data. For example, coupled dictionaries have been successfully used for describing the connection between high- and low-resolution features in image super-resolution [17]. Similarly, one can use CDL to capture blurred and focused image features [14]. In the context of multi-focus image fusion, CDL can be formulated as the following minimization problem:

$$
\begin{aligned}
& \min _{\boldsymbol{D}_{F}, \boldsymbol{D}_{B}, \boldsymbol{A}}\left\|\boldsymbol{X}_{\mathrm{Ft}}-\boldsymbol{D}_{F} \boldsymbol{A}\right\|_{\mathcal{F}}^{2}+\left\|\boldsymbol{X}_{\mathrm{Bt}}-\boldsymbol{D}_{B} \boldsymbol{A}\right\|_{\mathcal{F}}^{2} \\
& \text { s.t. }\left\|\boldsymbol{\alpha}_{i}\right\|_{0} \leqslant K,\left\|\left[\boldsymbol{d}_{\mathrm{Ft}}\right]_{j}\right\|_{2}=1,\left\|\left[\boldsymbol{d}_{\mathrm{Bt}}\right]_{j}\right\|_{2}=1, \forall j, i
\end{aligned}
$$

where $\boldsymbol{D}_{\mathrm{F}} \in \mathbb{R}^{n \times q}$ and $\boldsymbol{D}_{\mathrm{B}} \in \mathbb{R}^{n \times q}$ represent the focused and blurred dictionaries, respectively, with $\left[\boldsymbol{d}_{\mathrm{F}}\right]_{j},\left[\boldsymbol{d}_{\mathrm{B}}\right]_{j}$ referring to their $j$-th columns (i.e., atoms). The focused and blurred training data are denoted as $\boldsymbol{X}_{\mathrm{Ft}}$ and $\boldsymbol{X}_{\mathrm{Bt}}$, respectively. The $i$-th column of the common sparse representation matrix $\boldsymbol{A}$ is denoted as $\boldsymbol{\alpha}_{i}$. Finally, $\|\cdot\|_{0}$ is the operator counting the number of non-zero coefficients in a vector, $K$ is the maximum number of such coefficients, and $\|\cdot\|_{\mathcal{F}}$ denotes the Frobenius norm. In image fusion, CDL can be interpreted as an approximation of the underlying blurring function in the form of a linear transformation between any two tight column-wise corresponding subspaces of $\boldsymbol{D}_{\mathrm{F}}$ and $\boldsymbol{D}_{\mathrm{B}}$. Once the coupled dictionaries are learned, the joint sparse representation of two input image patches can be used for reconstruction or for building a fusion rule, as will be explained later in this paper.

In this work, we introduce a novel CDL-based image fusion method. The proposed approach consists of two main stages. First, CDL is used to capture the relationships between blurred 
and focused features by learning coupled dictionaries from labelled training data. In order to improve the discriminability of the dictionaries, a CDL method using structured incoherency is proposed. In a second stage, the reconstruction errors (obtained after a sparse coding phase) of a pair of input patches are used to identify the focused patch. In contrast to the method of [14], we do not separate the sparse representations in each dictionary, but rather promote the correlation between blurred and focused images by enforcing joint sparsity. Furthermore, we employ the reconstruction errors instead of the sparsitylevel as a discriminative rule. Finally, an all-in-focus image is obtained by averaging the selected focused patches. The effect of blocking artefacts is mitigated by applying a sliding window approach. Since the patches located between blurred and focused areas contain varying focus levels, we propose a pixelwise strategy for handling boundary regions. Experimental results using two natural image datasets and a sequence of in vivo microscopy images are presented in Section III The results show the proposed method to be more effective than several existing fusion approaches.

\section{IMAGE FUSION USING CDL}

\section{A. Problem formulation}

We consider the fusion problem where, for simplicity but without loss of generality, two images $\boldsymbol{I}_{1} \in \mathbb{R}^{N \times M}$ and $\boldsymbol{I}_{2} \in$ $\mathbb{R}^{N \times M}$ with varying focus levels are fused into an all-in-focus image $\boldsymbol{Y} \in \mathbb{R}^{N \times M}$. We propose to use a patch-wise approach where $n_{p}$ patches of size $n$ are extracted from $\boldsymbol{I}_{1}$ and $\boldsymbol{I}_{2}$, then concatenated into two matrices $\boldsymbol{X}_{1} \in \mathbb{R}^{n \times n_{p}}$ and $\boldsymbol{X}_{2} \in$ $\mathbb{R}^{n \times n_{p}}$, respectively. The corresponding single input patches are denoted by $\boldsymbol{x}_{1} \in \mathbb{R}^{n}$ or $\boldsymbol{x}_{2} \in \mathbb{R}^{n}$. After selecting the focused patches $\boldsymbol{x}_{F}$ from the pair $\left(\boldsymbol{x}_{1}, \boldsymbol{x}_{2}\right)$ and concatenating them into a matrix $\boldsymbol{X}_{F} \in \mathbb{R}^{n \times n_{p}}$, the final all-in-focus image is obtained by weighted averaging as follows

$$
\boldsymbol{Y}=\mathcal{P}^{*}\left(\boldsymbol{X}_{F}\right),
$$

where $\mathcal{P}(\cdot): \mathbb{R}^{N \times N} \mapsto \mathbb{R}^{n \times n_{p}}$ is a linear operator that extracts $n_{p}$ overlapping patches of size $n$ from an image, and $\mathcal{P}^{*}(\cdot)$ is its adjoint operation, which places each patch into its location in the image and performs averaging depending on the amount of overlap between patches, i.e., $\quad \mathcal{P}^{*}[\mathcal{P}(\boldsymbol{I})]=\boldsymbol{I}$. The following subsections first provide some details on the CDL approach used for learning the dictionaries $\boldsymbol{D}_{\mathrm{F}}$ and $\boldsymbol{D}_{\mathrm{B}}$. Then the sparse representation-based selection rule allowing the patches $\boldsymbol{x}_{F}$ to be selected is presented.

\section{B. $C D L$ with structured incoherency}

Prior to fusion, two coupled dictionaries $D_{F}$ (focused) and $\boldsymbol{D}_{\mathrm{B}}$ (blurred) are learned using labelled training data. The dictionaries are obtained by solving (1), as explained in Section II In this work, we choose to solve (1) using the algorithm proposed in [22]. More specifically, the method of [22] is based on an iterative minimization approach, that alternates between minimizations with respect to the dictionaries $D_{\mathrm{F}}$ and $\boldsymbol{D}_{\mathrm{B}}$, and the sparse codes in $\boldsymbol{A}$. The sparse coding step is solved using the orthogonal matching pursuit (OMP)
[19], while the dictionary update is obtained by solving the following minimization problem:

$$
\left[\boldsymbol{d}_{c}\right]_{j}=\underset{\left[\boldsymbol{d}_{c}\right]_{j}}{\operatorname{argmin}}\left\|\left[\boldsymbol{E}_{c}\right]_{j}-\left[\boldsymbol{d}_{c}\right]_{j}\left[\boldsymbol{\alpha}_{j}^{T}\right]_{\boldsymbol{J}_{j}}\right\|_{\mathcal{F}}^{2}, c \in\{\mathrm{F}, \mathrm{B}\},
$$

such that

$$
\left[\boldsymbol{E}_{c}\right]_{j} \triangleq\left[\boldsymbol{X}_{c}-\sum_{s \neq j}\left[\boldsymbol{d}_{c}\right]_{s} \boldsymbol{\alpha}_{j}^{T}\right]_{\boldsymbol{J}_{j}} \text { and } \boldsymbol{f}_{\boldsymbol{j}}=\left\{i \mid\left[\boldsymbol{\alpha}_{j}^{T}\right]_{i} \neq 0\right\}
$$

where $\boldsymbol{\alpha}_{j}^{T}$ is the $j$ th row of $\boldsymbol{A}, \boldsymbol{J}_{\boldsymbol{j}}$ is an indicator function that selects the non-zero entries in $\boldsymbol{\alpha}_{j}^{T}$, and the subscript $c$ stands for the labels $\mathrm{F}$ (for focused) or B (for blurred training data). Since the dictionaries are used to classify the patches as either belonging to class F or B (see Subsection II-C), it is desirable that the dictionaries provide the best discriminative power. In this work, a discriminative constraint based on structured incoherency is added to (3). Note that this approach has been successfully used in [21] for the discriminative dictionary learning. The key idea is to add a constraint that enforces each dictionary to be weak at representing other classes. The incoherency term takes the form $\mathcal{C}\left(\boldsymbol{D}_{\mathrm{F}}, \boldsymbol{D}_{\mathrm{B}}\right)=\left\|\boldsymbol{D}_{\mathrm{F}}^{T} \boldsymbol{D}_{\mathrm{B}}\right\|_{\mathcal{F}}^{2}$. The dictionary update problem becomes

$$
\begin{gathered}
{\left[\boldsymbol{d}_{c}\right]_{j}=\underset{\left[\boldsymbol{d}_{c}\right]_{j}}{\operatorname{argmin}}\left\|\left[\boldsymbol{E}_{c}\right]_{j}-\left[\boldsymbol{d}_{c}\right]_{j}\left[\boldsymbol{\alpha}_{j}^{T}\right]_{\boldsymbol{f}_{j}}\right\|_{\mathcal{F}}^{2}+\lambda\left\|\boldsymbol{D}_{h}^{T}\left[\boldsymbol{d}_{c}\right]_{j}\right\|_{2}^{2}} \\
c \in\{\mathrm{F}, \mathrm{B}\}, h=\{\mathrm{F}, \mathrm{B}\}-c,
\end{gathered}
$$

where $\lambda>0$ controls the trade-off between the reconstructive and discriminative properties of the dictionaries $\boldsymbol{D}_{\mathrm{F}}$ and $\boldsymbol{D}_{\mathrm{B}}$. Note that in (4), the incoherency term is formulated atomwise. The corresponding atom update rule is then formulated as follows:

$$
\left[\boldsymbol{d}_{c}\right]_{j}=\left(\lambda \boldsymbol{D}_{h} \boldsymbol{D}_{h}^{T}+\left\|\boldsymbol{\alpha}_{j}^{T}\right\|_{2}^{2} \boldsymbol{I}_{\boldsymbol{d}}\right)^{-1}\left[\boldsymbol{E}_{c}\right]_{j}\left[\boldsymbol{\alpha}_{j}^{T}\right]_{\boldsymbol{j}_{j}}^{T}, c \in\{\mathrm{F}, \mathrm{B}\}
$$

where $\boldsymbol{I}_{\boldsymbol{d}}$ denotes the identity matrix. For a more detailed description of the remaining steps of the CDL algorithm, the reader is referred to [22].

\section{Fusion using sparse representation}

A classical way of classifying input signals using sparse representation is by evaluating their reconstruction errors [20]. The key idea is that each element should be assigned to the class providing the smallest reconstruction error, i.e., the best sparse representation. However, since multi-focus images are highly correlated, standard dictionary learning methods lead to a considerable overlap between the dictionaries $\boldsymbol{D}_{\mathrm{F}}$ and $\boldsymbol{D}_{\mathrm{B}}$, making classification impractical. The CDL framework described in Subsection $\amalg-\mathrm{B}$ allows to overcome this issue by insuring that the learnt dictionaries are sufficiently independent and discriminative. In particular, classification can be performed using a concatenation of the coupled dictionaries $\left[\begin{array}{ll}\boldsymbol{D}_{\mathrm{F}}^{T} & \boldsymbol{D}_{\mathrm{B}}^{T}\end{array}\right]^{T}$ and $\left[\begin{array}{ll}\boldsymbol{D}_{\mathrm{B}}^{T} & \boldsymbol{D}_{\mathrm{F}}^{T}\end{array}\right]^{T}$. These matrices can be used as means of describing the function between two patches $\boldsymbol{x}_{1}$ and $\boldsymbol{x}_{2}$, i.e., blurring and deblurring, respectively. Note that since identifying focused patches is a binary classification problem, it is sufficient to use one dictionary $\boldsymbol{D} \triangleq\left[\begin{array}{ll}\boldsymbol{D}_{\mathrm{F}}^{T} \boldsymbol{D}_{\mathrm{B}}^{T}\end{array}\right]^{T}$. 
1) Selection rule: In this work, the focused patches $\boldsymbol{x}_{F}$ are selected using the reconstruction error-based approach. More specifically, let $e_{1}$ and $e_{2}$ be the reconstruction errors associated with the blurring and deblurring functions, respectively. This can be formulated as

$$
\left\{\begin{aligned}
e_{1} & =\left\|\left[\begin{array}{ll}
\boldsymbol{x}_{1}^{T} & \boldsymbol{x}_{2}^{T}
\end{array}\right]^{T}-\boldsymbol{D} \boldsymbol{\alpha}\right\|_{2}^{2} \\
e_{2} & =\left\|\left[\begin{array}{ll}
\boldsymbol{x}_{2}^{T} & \boldsymbol{x}_{1}^{T}
\end{array}\right]^{T}-\boldsymbol{D} \boldsymbol{\alpha}\right\|_{2}^{2}
\end{aligned}\right.
$$

where $\boldsymbol{\alpha}$ contains the associated sparse codes. The selection rule is then based on the fact that a relatively smaller value of $e_{1}$ indicates that $\boldsymbol{x}_{1}$ has undergone blurring (resulting in $\boldsymbol{x}_{2}$ ). For the purpose of processing the boundary region (explained below), a pixel-wise sparse representation error is also defined as follows:

$$
\left\{\begin{array}{l}
\boldsymbol{e}_{\mathbf{1}}^{\prime}=\left(\boldsymbol{x}_{1}-\boldsymbol{D}_{\mathrm{F}} \boldsymbol{\alpha}\right)^{2}+\left(\boldsymbol{x}_{2}-\boldsymbol{D}_{\mathrm{B}} \boldsymbol{\alpha}\right)^{2} \\
\boldsymbol{e}_{\mathbf{2}}^{\prime}=\left(\boldsymbol{x}_{1}-\boldsymbol{D}_{\mathrm{B}} \boldsymbol{\alpha}\right)^{2}+\left(\boldsymbol{x}_{2}-\boldsymbol{D}_{\mathrm{F}} \boldsymbol{\alpha}\right)^{2}
\end{array}\right.
$$

The selection rule for the pair of patches $\boldsymbol{x}_{1}$ and $\boldsymbol{x}_{2}$ can then be expressed as

$$
\left\{\begin{array}{lr}
\boldsymbol{x}_{F}=\boldsymbol{x}_{1}, & \text { if } e_{1}<e_{2} \\
\boldsymbol{x}_{F}=\boldsymbol{x}_{2}, & \text { otherwise }
\end{array}\right.
$$

Note that in $(8)$ the equality condition is not taken into account since $e_{1}$ and $e_{2}$ cannot be equal.

2) Fusion and processing of boundary regions: Once the selection rule is applied to all the patches in the images, a patch-wise decision mask $\boldsymbol{M}_{p}$ is straightforwardly obtained (such that the patches in $\boldsymbol{M}_{p}$ contain values $c \in\{1,2\}$ ). The pixel-wise decision mask is reconstructed using

$$
M=\mathcal{P}^{*}\left(M_{p}\right)
$$

where $\boldsymbol{M}$ now contains values $c^{\prime} \in[1,2]$. One could directly use $\boldsymbol{M}$ to form the final image $\boldsymbol{Y}$ by assigning pixels according to their labels at each location. However, this approach can lead to errors around the boundaries between blurred and focused regions, caused by the weighted averaging of patches containing both blurred and focused pixels. In order to bypass this issue, a pixel-wise labelling is proposed for these regions. More specifically, the mask $M$ is used to first detect the boundary region, which contains all the pixels with labels in the interval $] 1,2[$. In the second step, the pixel-wise sparse coding errors (7) are used to assign new labels to the pixels within the boundary region ${ }^{1}$ Finally, the all-in-focus image $\boldsymbol{Y}$ is obtained by assigning pixels according to their labels in $M$ to each location $(k, l)$, as follows:

$$
\boldsymbol{Y}_{k l}=\left(2-\boldsymbol{M}_{k l}\right) \boldsymbol{I}_{1, k l}+\left(\boldsymbol{M}_{k l}-1\right) \boldsymbol{I}_{2, k l}
$$

The different steps of the proposed image fusion algorithm are summarized in Algorithm 1

\section{EXPERIMENTS}

In this section, the proposed method is compared with four recent fusion methods including state-of-the-art. Specifically,

${ }^{1}$ Figure 2 $\mathrm{c}$ shows an example of a mask obtained using the proposed approach.

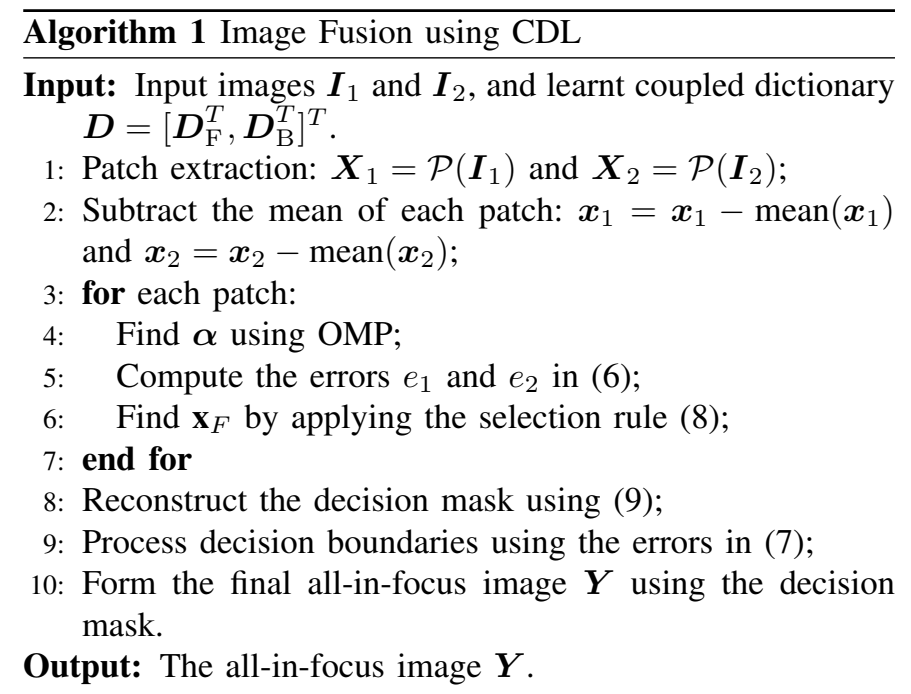

these methods include one transform domain approach using Laplacian pyramids (LP) [28], a spatial domain method using phase congruency (PC) [9], a sparse representationbased approach using convolutional dictionaries (CSR) [29], and a method for microscopic medical image fusion using mean-shift segmentation [32]. For the data without available ground-truth, the evaluation is based on the normalized mutual information (NMI) [26], the objective image fusion performance measure $\left(Q_{A B / F}\right)[25]$ and Tone mapped index (TMQI) [27]. When ground-truth is available, we also use the mean-squared-error (MSE). First, experiments are conducted on two widely used multi-focus datasets referred to as Lytro [23] and Grayscale [24]. Note that the Grayscale dataset provides ground-truth all-in-focus images, which can be used to compute the MSE. In a second experiment, a sequence of real medical images is used to validate the proposed method. Specifically, a sequence of 15 partially blurred and noisy microscopy images is used [31]. All experiments are performed on a PC running an Intel(R) Xeon(R) 3.40GHz CPU.

The coupled dictionaries $\boldsymbol{D}_{\mathrm{F}}$ and $\boldsymbol{D}_{\mathrm{B}}$ are learnt using 40000 pairs of blurred and focused patches extracted from 4 images

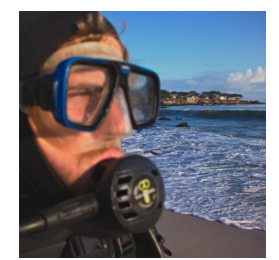

(a)

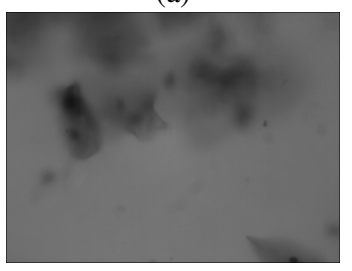

(c)

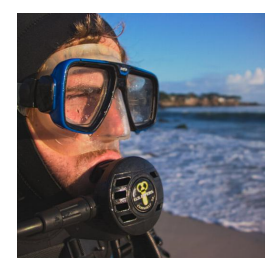

(b)

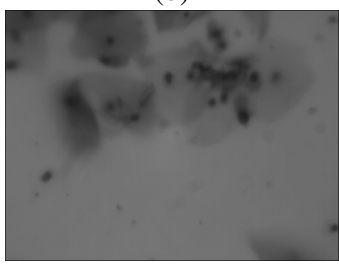

(d)
Fig. 1: Examples of multi-focus images from the Lytro dataset $(a, b)$ and the microscopic medical image sequence $(c, d)$. 
of the Lytro dataset (the rest of the images are used for testing). The dictionary learning parameters are set empirically with a sparsity parameter $K=10$, a redundancy of 4 and a patch size of $16 \times 16$. The resulting dictionaries are of size $256 \times 1024$. Finally, the maximum number of iterations of the CDL step is set to 20 .

\section{A. Fusion results using Lytro and Grayscale images}

The fusion results are quantitatively compared in terms of average NMI, $Q_{A B / F}$, and MSE for the two considered datasets. The results reported in Table. I show that the best overall performance is provided by the proposed and PC methods for both datasets. More specifically, the PC and proposed approaches lead to similar NMI and $Q_{A B / F}$ values, while the proposed method results in significantly lower MSE. One can also see that the proposed method provides competitive execution times.

Fig. 2 shows the resulting all-in-focus images obtained for the input images in Fig. 1 - $(a, b)$ using the proposed and PC methods. Note that the mask is first computed for the grayscale version of the images before applying it to the RGB layers. A visual inspection of the resulting all-in-focus images shows that the proposed method allows the edges to be preserved. In particular, the boundaries between blurred and focused regions are sharper, as can be clearly observed in the magnified regions in Fig. 2.d and Fig. 2.-e.

\begin{tabular}{|c|c|c|c|c|c|c|}
\hline & Dataset & $\boldsymbol{Q}_{\boldsymbol{A B} / \boldsymbol{F}}$ & $\boldsymbol{N} \boldsymbol{M I}$ & $\boldsymbol{T M Q I}$ & $\boldsymbol{M S E}$ & $\begin{array}{c}\text { Execution } \\
\text { time (s) }\end{array}$ \\
\hline \multirow{2}{*}{$\mathbf{L P}$} & Gray & 0.7434 & 1.0406 & 0.9347 & 6.0308 & 0.0056 \\
& Lytro & 0.7524 & 1.0306 & 0.6628 & - & 0.0107 \\
\hline \multirow{2}{*}{$\mathbf{P C}$} & Gray & 0.7535 & 1.2216 & 0.9321 & 6.8984 & 0.5104 \\
& Lytro & 0.7397 & 1.2089 & 0.6648 & - & 1.0557 \\
\hline \multirow{2}{*}{ CSR } & Gray & 0.7198 & 1.0296 & 0.9292 & 4.6927 & 54.8437 \\
& Lytro & 0.7304 & 1.0340 & 0.6619 & - & 99.1661 \\
\hline \multirow{2}{*}{ Us } & Gray & 0.7512 & 1.1772 & 0.9331 & 3.3453 & 1.4180 \\
& Lytro & 0.7561 & 1.1913 & 0.6628 & - & 2.5627 \\
\hline
\end{tabular}

TABLE I: The average results of NMI, $Q_{A B / F}$, TMQI, MSE and execution time for the Lytro and Grayscale datesets.

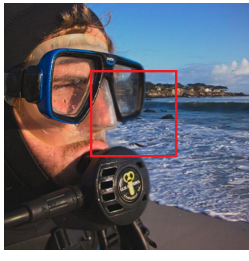

(a)

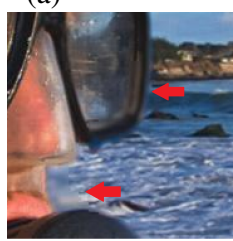

(d)

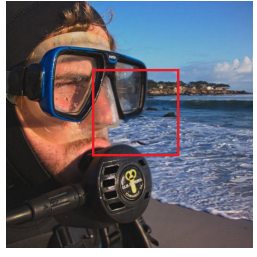

(b)

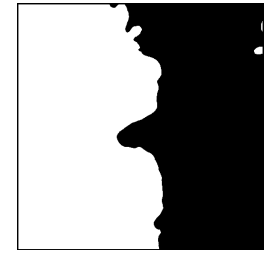

(c)

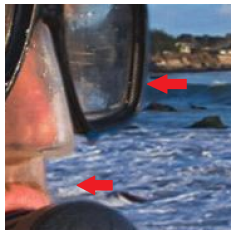

(e)
Fig. 2: Fusion results by (a) PC and (b) the proposed method, the corresponding magnified regions $(\mathrm{d}, \mathrm{e})$, and (c) the mask obtained using the proposed method.

\section{B. Fusion of in vivo Pap Smear images}

Papanicolaou test (Pap smear) images are used for the automated diagnosis of cervical cancer. However, these images are characterized by the presence of different focus levels due to the limited depth of field of the microscope. In order to bypass this limitation, multiple images with different focal settings are acquired and fused into one all-in-focus image [30]. In this work, we use a sequence of 15 multi-focus Pap smear images (of size $480 \times 640$ pixels). The sequence is processed using a single-elimination approach. This means that the fusion is conducted sequentially and pair-wise, i.e., the fusion result from each pair of images is in turn fused with the next input image. The quantitative evaluation of the results obtained using the proposed method, PC, and the method of [32] are summarized in Table. II] The resulting fused images are displayed in Fig. 3 .

The results reported in Table. III show that the proposed method provides higher $Q_{A B / F}$ and NMI values, corresponding to more edge information and a higher fidelity of pixel intensities. Fig. 3 confirms these findings, as one can see that the proposed method provides sharper edges (green arrows) and preserves details (red arrows) that are missing in the image obtained by the method of [32].

\begin{tabular}{|c|c|c|c|}
\hline & $\boldsymbol{Q}_{\boldsymbol{A B} / \boldsymbol{F}}$ & $\boldsymbol{N} \boldsymbol{M I}$ & $\boldsymbol{T M Q I}$ \\
\hline The method of $\mathbf{3 2}$ & 0.6063 & 5.9135 & 0.7949 \\
\hline PC & 0.6212 & 6.9133 & 0.7739 \\
\hline The proposed method & 0.6257 & 7.1941 & 0.7755 \\
\hline
\end{tabular}

TABLE II: Fusion performance for the Pap smear images.

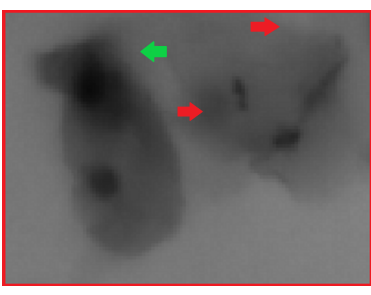

(a)

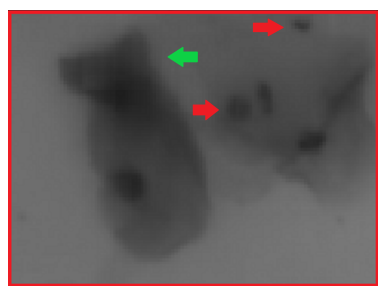

(b)
Fig. 3: Magnified region in the fused image obtained by (a) the method of [32], and (b) the proposed method.

\section{CONCLUSION}

This paper has introduced an image fusion algorithm using coupled dictionary learning and joint sparse representation. First, a coupled dictionary learning approach with an additional incoherency constraint has been used to learn blurred and focused dictionaries. Secondly, the patch-wise sparse representation errors have been used to construct a fusion rule for input patches with unknown focus levels. In addition, a pixel-wise processing of the boundary regions has been proposed. Experiments have been conducted using two natural image datasets and a sequence of in vivo microscopic images (Pap smear). A comparison with state-of-the-art approaches has shown the competitiveness of the proposed method in terms of various image fusion metrics. 


\section{REFERENCES}

[1] M. Subbarao, T. Choi, and A. Nikzad, "Focusing techniques," Opt. Eng., vol. 32, pp. 2824-2836, Mar. 1993.

[2] M. Born and E. Wolf, Principles of Optics. Cambridge Univ. Press., 1999.

[3] Q. Zhang, and B. L. Guo, "Multifocus image fusion using the nonsubsampled contourlet transform," Signal Process., vol. 89, pp. 1334-1346, Jul. 2009.

[4] F. Nencini, A. Garzelli, S. Baronti, and L. Alparone, "Remote sensing image fusion using the curvelet transform," Inf. Fusion, vol. 8, no. 2, pp. 143-156, Apr. 2007.

[5] G. Pajares and J. Cruz, "A wavelet-based image fusion tutorial," Pattern Recognit., vol. 37, no. 9, pp. 1855-1872, Sep. 2004

[6] O. Rockinger, "Image sequence fusion using a shift-invariant wavelet transform," in Proc. IEEE Int. Conf. Image Process., Santa Barbara, CA, 1997, pp. 288-291.

[7] V. D. Calhoun and T. Adali, "Feature-based fusion of medical imaging data," in IEEE Trans. Inf. Technol. Biomedicine, vol. 13, no. 5, pp. 711720, Sep. 2009.

[8] L. Cao, L. Jin, H. Tao, G. Li, Z. Zhuang, and Y. Zhang, "Multi-Focus Image Fusion Based on Spatial Frequency in Discrete Cosine Transform Domain," in IEEE Signal Processing Letters, vol. 22, no. 2, pp. 220-224, Sep. 2014.

[9] K. Zhan, Q. Li, J. Teng, M. Wang, and J. Shi, "Multifocus image fusion using phase congruency," in Journal of Electronic Imaging, vol. 24 no. 3, pp. 0330141-03301412, May. 2015.

[10] H. Li, L. Li, and J. Zhang, "A novel DWT based multi-focus image fusion method," in Procedia Eng., vol. 24, pp. 177-181, 2011.

[11] B. Yang and S. Li, "Multifocus image fusion and restoration with sparse representation," in IEEE Trans. Instrum. Meas., vol. 59, no. 4, pp. 884 892, Apr. 2010.

[12] M. Nejati, S. Samavi, and S. Hirani, "Multi-focus image fusion using dictionary-based sparse representation," in Inf. Fusion, vol. 25, pp. 7284, Sep. 2015

[13] Q. Zhang, and M. D. Levine, "Robust multi-focus image fusion using multi-task sparse representation and spatial context," IEEE Trans. Image Process., vol. 25, no. 5, pp. 2045-2058, Mar. 2016.

[14] R. Gao, S. A. Vorobyov, and H. Zhao, "Multi-focus image fusion via coupled dictionary training," in Proc. IEEE Int. Conf. Acoustics, Speech and Signal Process., Shanghai, China, 2016, pp. 1666-1670.

[15] J. Yang, J. Wright, T. S. Huang, and Y. Ma, "Image super-resolution via sparse representation," IEEE Trans. Image Process., vol. 19, no. 11, pp. 2861-2873, May. 2010.

[16] T. Peleg, and M. Elad, "A statistical prediction model based on sparse representations for single image super-resolution," IEEE Trans. Image Process., vol. 23, no. 6, pp. 2569-2582, Jun. 2014.

[17] J. Yang, Z. Wang, Z. Lin, S. Cohen, and T. Huang, "Coupled dictionary training for image super-resolution," IEEE Trans. Image Process. vol. 21, no. 8, pp. 3467-3478, Aug. 2012.

[18] S. Wang, L. Zhang, Y. Liang, and Q. Pan, "Semi-coupled dictionary learning with applications to image super-resolution and photo-sketch synthesis," in Proc. IEEE Int. Conf. Comput. Vis. Pattern Recognit., Rhode Island, USA, 2012, pp. 2216-2223.

[19] J. A. Tropp, and A.C. Gilbert,"Signal recovery From random measurements via orthogonal matching pursuit," in Proc. IEEE Trans. Inf. Proc., vol. 53, no. 12, pp. 4655-4666, Dec. 2007.

[20] K. Skretting, and J. H. Husoy, " Texture classification using sparse framebased representations," in EURASIP Journal on Advances in Signal Processing, vol. 2006, pp. 102-112, 2006.

[21] I. Ramirez, P. Sprechmann, and G. Sapiro,"Classification and clustering via dictionary learning with structured incoherence and shared features," in IEEE Computer Society Conference on Computer Vision and Pattern Recognition, San Francisco, CA, USA, pp. 3501-3508, Jun. 2010.

[22] F. G. Veshki, and S. A. Vorobyov, "An Efficient Coupled Dictionary Learning Method," in IEEE Signal Processing Letters, vol. 26, no. 10, pp. 1441-1445, Aug. 2019.

[23] M. Nejati, S. Samavi, and S. Hirani, "Lytro Multi Focus Dataset," [Online]. Available: http://mansournejati.ece.iut.ac.ir/content/ lytro-multi-focus-dataset [Accessed Oct. 16, 2019].

[24] J. Saeedi, K. Faez, "Multi Focus Image Dataset," [Online]. Available: https://www.researchgate.net/profile/Jamal_ Saeedi/publication/273000238_multifocus_image_dataset/data/ 54f489b80cf2ba6150635697/multi-focus-image-dataset.rar [Accessed Oct. 16, 2019].

[25] C. Xydeas and V. Petrović, "Objective image fusion performance measure," Electron. Lett., vol. 36, no. 4, pp. 308-309, Feb. 2000.
[26] M. Hossny, S. Nahavandi and D. Creighton, "Comments on Information measure for performance of image fusion," Electron. Lett., pp. 10661067, 2008.

[27] H. Yeganeh, and Z. Wang, "Objective Quality Assessment of Tone Mapped Images," IEEE Transactios on Image Processing, vol. 22, no. 2, pp. 657-667, Feb. 2013.

[28] W. Wang and F. Chang, "A Multi-focus Image Fusion Method Based on Laplacian Pyramid," Journal of Computers, vol. 6, no. 12, pp. 25592566, 2011.

[29] Y. Liu, X. Chen, R. K. Ward and Z. J. Wang, "Image Fusion With Convolutional Sparse Representation," in IEEE Signal Processing Letters, vol. 23 , no. 12 , pp. 1882-1886, Dec. 2016.

[30] J. Tian and L. Chen, "Adaptive multi-focus image fusion using a waveletbased statistical sharpness measure," in Signal Processing, vol. 92, no. 9, pp. 2137-2146, 2012.

[31] S. Tello-Mijares, "Multi focus image fusion," [Online]. Available: https://drive.google.com/drive/folders/ 1bcrJM8Kw0mzKF8VKpHg1WVlOxWGulwdj] [Accessed Feb. 2, 2020].

[32] S. Tello-Mijares and J. Bescos, "Region-based multifocus image fusion for the precise acquisition of Pap smear images," in Journal of Biomedical Optics, vol. 23, no. 5, pp. ()056005)1-9, May. 2018. 